\title{
Weak probe readout of coherent impurity orbital superpositions in silicon
}

\author{
K. L. Litvinenko, ${ }^{1}$ P. T. Greenland, ${ }^{2}$ B. Redlich, ${ }^{3}$ C. R. Pidgeon, ${ }^{4}$ G. Aeppli,${ }^{5,6,7}$ and B. N. Murdin ${ }^{1}$ \\ ${ }^{1}$ Advanced Technology Institute and SEPNet, University of Surrey, Guildford, GU2 7XH, United Kingdom \\ ${ }^{2}$ London Centre for Nanotechnology and Department of Physics and Astronomy, University College London, \\ London WC1H OAH, United Kingdom \\ ${ }^{3}$ Radboud University, Institute for Molecules and Materials, FELIX Laboratory, Toernooiveld 7c, 6525 ED Nijmegen, Netherlands \\ ${ }^{4}$ Institute of Photonics and Quantum Science, SUPA, Heriot-Watt University, EH14 4AS, United Kingdom \\ ${ }^{5}$ Laboratory for Solid State Physics, ETH Zurich, Zurich, CH-8093, Switzerland \\ ${ }^{6}$ Institut de Physique, EPF Lausanne, Lausanne CH-1015, Switzerland \\ ${ }^{7}$ Swiss Light Source, Paul Scherrer Institut, Villigen PSI, CH-5232, Switzerland
}

(Received 12 August 2016; published 20 December 2016)

\begin{abstract}
Pump-probe spectroscopy is the most common time-resolved technique for investigation of electronic dynamics, and the results provide the incoherent population decay time $T_{1}$. Here we use a modified pump-probe experiment to investigate coherent dynamics, and we demonstrate this with a measurement of the inhomogeneous dephasing time $T_{2}^{*}$ for phosphorus impurities in silicon. The pulse sequence produces the same information as previous coherent all-optical (photon-echo-based) techniques but is simpler. The probe signal strength is first order in the pulse area but its effect on the target state is only second order, meaning that it does not demolish the quantum information. We propose simple extensions to the technique to measure the homogeneous dephasing time $T_{2}$, or to perform tomography of the target qubit.
\end{abstract}

DOI: 10.1103/PhysRevB.94.235207

\section{INTRODUCTION}

Silicon acts as an atom trap for dopants such as phosphorus [1], and as for atoms in traps, the extra electrons (of the dopant relative to the silicon host) occupy a hydrogenlike series of orbitals. The Rydberg energy is renormalized strongly downwards in proportion to $m^{*} / \varepsilon_{r}^{2}$ where $\varepsilon_{r}$ is the dielectric constant of silicon which is approximately ten times larger than in vacuum, and $m^{*}$ is the effective mass of conduction electrons which is roughly five times smaller in silicon than in vacuum. The electric dipole transitions are associated with the orbital quantum number and thus occur in the $\mathrm{THz}$ frequency range. They have been a topic of long-standing interest in semiconductor physics, finding applications as probes of impurity environments [2-4] and, most recently, even the degree of spin polarization of the ground state [5]. Coherent $\mathrm{THz}$ control of excited orbitals of phosphorus impurities in silicon is also potentially useful for the control of magnetic exchange interactions between impurity spins [6]. Here we are concerned only with orbital states and electric dipole transitions between them. Key to the ultimate utility of such control is knowledge of state evolution after a coherent $\mathrm{THz}$ pulse. Relevant processes are random phase jumps (homogeneous dephasing with time scale $T_{2}$ ), phase loss by oscillators evolving at different rates due to different natural frequencies (inhomogeneous dephasing, $\left.T_{2}^{*}\right)$, and energy relaxation $\left(T_{1}\right)$.

For quantum measurements and control, time-domain pump-probe experiments are indispensable and have been enabled by the development of free-electron lasers producing transform-limited THz pulses. Various pump-probe techniques measure the different dynamics of the polarization and population as a function of time after the pump (control) pulse. For example, transmission of a probe pulse at an angle to the pump can reveal $T_{1}$ [1]. Alternatively, controlled rephasing of inhomogeneous phase loss by a time-reversal pulse can produce a photon echo whose strength depends on $T_{2}$ [7].
In a Ramsey interference experiment the coherence produced by the pump pulse can be either enhanced or destroyed by a second pulse depending on their phase difference, and the envelope of the fringes reveals $T_{2}^{*}$ [8]. In each of these cases frequency-domain experiments can be used to obtain the same information, but time-domain versions can be preferable when there are multiple processes responsible for the decays as well as static, inhomogeneous broadening effects that must be separated from dynamics. Furthermore, pulsed experiments are a requirement for demonstration of coherent control and state readout of qubits. In this work we describe a pulsed method of qubit readout that preserves the phase and amplitude to first order, and we apply the technique to phosphorus-doped silicon, an important quantum technology platform.

THz-pumped orbital excitations of silicon impurities have been controlled and detected both incoherently and coherently to provide various dynamical time scales $T_{1}[1,8,9]$ (including the ionized donor recombination rate [10] and the interdonor tunneling rate [11]), $T_{2}$ [7], and $T_{2}^{*}[8,12]$. The Bloch sphere simply maps the population (given by the longitudinal, $z$ component of the Bloch vector) and polarization (given by the transverse, $x-y$ component). $T_{1}$ describes the longitudinal relaxation, while $T_{2}$ and $T_{2}^{*}$ describe transverse relaxation. It is typical to use incoherent readout of the incoherent decay $[1,9,10]$ and coherent readout of the coherent decay $[7,8]$, but it is also possible to utilize coherent readout of incoherent dynamics, as in the example of echo detection of $T_{1}$ [8], and incoherent readout of coherent dynamics, as in electrical detection of Ramsey interference [8,12]. In the latter the electrical signal arises from thermal ionization of excited orbital states, and it measures the excited state population. In the case of an electrical Ramsey experiment the polarization left by the first pulse is projected onto the $z$ axis of the Bloch sphere by the second pulse, ready for readout. The electrical readout is at least one order of magnitude more sensitive than the coherent optical echo detection. Another advantage of 
electrical readout is that (if thermal ionization is replaced by controlled resonant tunneling through the barrier of a single electron transistor) it may be incorporated for readout of single orbital qubits in existing quantum computing schemes [13]. The disadvantage of electrical readout is that it requires very careful calibration to produce information on the fractional population difference, to confirm that the current is linear with population and to establish the proportionality constant [12]. For an optical experiment it is in principle possible to measure the fractional probe absorption relatively easily.

In this work we use an incoherent optical readout of the coherent dynamics based on the transmission of a weak probe beam, at an angle to the pump. In contrast with coherent echo detection, an incoherent pump-probe optical readout requires a much less sensitive optical arrangement. The data we produce here are similar in terms of signal to noise compared to that of the echo detection scheme used in Refs. [7,8], but were far easier to produce. Unlike electrical detection they yield an unambiguous measure of the projection of the Bloch vector without the need for careful calibration. Additionally, the probe has only second-order effects on the population of target states. This opens the possibility of introducing subsequent (or repeated) sequences, i.e., control pulse(s) and probe pulse(s), followed by further control pulse(s) and probe pulse(s). This is analogous to the aims of quantum nondemolition techniques, where the measured target particle may be repeatedly sensed with a "meter particle" without loss of the target particle's coherence allowing the possibility of further control or meter measurement [14]. The meter particle corresponds to the probe photon in our case.

In a pump-probe experiment a strong pump pulse excites the atoms. If the dephasing is fast compared with the pulse duration then the pump simply induces a bleaching of the probe transmission proportional to the population, and the angle between the beams is irrelevant. In our case, the pulses are short compared to the dephasing [1], which means that the interaction between the pump, atoms, and probe is coherent, and phase is therefore important. The angle between the pump and probe ensures that the phase between the atomic polarization and the probe varies over the sample volume. In the rotating frame defined by the pump, the atomic Bloch vectors are all rotated about the $x$ axis by an angle determined by the pulse area. The (weak) probe rotates them again by a (small) angle, and the axis of rotation is on the equator of the Bloch sphere at an azimuthal angle that varies with spatial position. As shown in Fig. 1(a), if the probe finds the Bloch vector on the equator, then after averaging over the interaction volume, it has no effect on the population. Otherwise, the effect of the probe averaged over the sample volume is to reduce the population, and the change is $\langle\Delta z\rangle=-s^{2} z / 4$ (see Supplemental Material [15]), where $z=n_{2}-n_{1}$ and $n_{1,2}$ are the populations in the ground and excited states, respectively. The probe pulse "area" $s=\Omega \tau$, where $\Omega=\mu F / \hbar$ is the Rabi frequency, $\tau$ is the pulse duration, $F$ is the electric field amplitude of the pulse, and $\mu$ is the atomic dipole moment. Thus for $n_{1}>n_{2}$ the atoms take energy from the probe beam (absorption), while for $n_{1}<n_{2}$ energy is given to the probe beam (stimulated emission). For a fully relaxed population $\left(n_{2}=0\right)$ the absorption is maximum. Therefore, the pump induces a reduction in the absorption (or even gain for a pulse

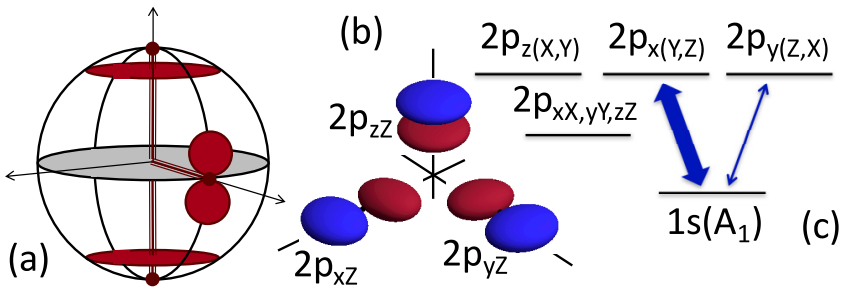

FIG. 1. (a) Bloch sphere representation of a two-level pumpprobe experiment with long $T_{2}$, when the probe is incident at an angle to the pump. The distribution of Bloch vectors produced after the probe pulse is shown (as red loops) when it (the probe) acts on a fully relaxed, a fully inverted, and a 50:50 superposition state. (b) The orbital excited states involved in the experiment. Each conduction band valley $(X, Y, Z)$ is characterized by a heavy effective mass along the valley axis $\left(m_{l}^{*}\right)$, and a light transverse mass $\left(m_{t}^{*}\right)$. The donor orbitals are mixed, but to a good approximation they are simply given by spherical harmonics with a coordinate scale factor of $\sqrt{m_{t}^{*} / m_{l}^{*}}(\sim 0.5$ for silicon) along the valley axis. The panel shows the $2 p$ states for the $Z$ valley. The mass anisotropy lifts the degeneracy: The $2 p_{0}$ state (i.e., the $2 p_{z}$ in the $Z$ valley) is lowered relative to the others. For zero magnetic field the $2 p_{ \pm}$states are degenerate, and for linearly polarized light they are more appropriately resolved into $2 p_{x, y}$. (c) The level scheme indicating the crossed-polarized pump $(x$-polarized) and probe ( $y$-polarized) transitions, forming a simple $\mathrm{V}$ scheme with a common ground state.

area $>\pi / 2$ ), and as the population relaxes towards the ground state after the pump pulse, the absorption recovers.

We now consider pump-probe experiments for a three-level scheme comprising the target qubit and a third, readout state [Fig. 1(c)]. The details are shown in the Supplemental Material [15]. In our example, the target qubit is formed by the ground $1 s$ state and the $2 p_{x}$ excited state, and is controlled by a linearly $x$-polarized laser pulse. The readout state is the $2 p_{y}$ excited state, which overlaps with the ground state for $y$-polarized light. The states form a three-level V scheme. For this scheme,

$$
\Psi=a_{1 s} \psi_{1 s}+a_{2 p x} \psi_{2 p x}+a_{2 p y} \psi_{2 p y},
$$

and the state vector A corresponding to Eq. (1) is

$$
\mathbf{A}=\left[\begin{array}{c}
a_{1 s} \\
a_{2 p x} \\
a_{2 p y}
\end{array}\right] .
$$

For atoms that start in the ground state,

$$
\mathbf{A}_{0}=\left[\begin{array}{l}
1 \\
0 \\
0
\end{array}\right],
$$

a single, $x$-polarized pump pulse of area $S$ transfers them to the state

$$
\mathbf{A}_{1}=\left[\begin{array}{c}
\cos \left(\frac{1}{2} S\right) \\
-i \sin \left(\frac{1}{2} S\right) \\
0
\end{array}\right]
$$


After a pair of equal such pulses, the atoms' state is found to be

$$
\mathbf{A}_{2}=\left[\begin{array}{c}
1-\frac{1}{2}(1-\cos S)\left(1+e^{i \omega t_{d}}\right) \\
-\frac{1}{2} i \sin S\left(1+e^{-i \omega t_{d}}\right) \\
0
\end{array}\right],
$$

where the frequency is $\omega$ and time delay is $t_{d}$. The population difference between $a_{1 s}$ and $a_{2 p x}$ is

$$
z=\left|a_{2 p x}\right|^{2}-\left|a_{1 s}\right|^{2}=\sin ^{2} S\left[1+\cos \left(\omega t_{d}\right)\right]-1,
$$

which clearly oscillates with pulse area (Rabi oscillations) and with $\omega t_{d}$ (Ramsey fringes).

As is clear from Eqs. (3)-(5) $x$-polarized control pulses leave the atom with $a_{2 p y}=0$, and following a weak probe pulse of area $s$ with $y$ polarization the state is

$$
\mathbf{B}_{\perp}=\left[\begin{array}{c}
\cos \left(\frac{1}{2} s\right) a_{1 s} \\
a_{2 p x} \\
-i \sin \left(\frac{1}{2} s\right) e^{-i \phi} a_{1 s}
\end{array}\right] .
$$

The probe absorption is proportional to the change in the difference in population of the $2 p_{y}$ and $1 s$ states, and is

$$
\begin{aligned}
\Delta \zeta & =\left|b_{2 p y}\right|^{2}-\left|b_{1 s}\right|^{2}-\left|a_{2 p y}\right|^{2}+\left|a_{1 s}\right|^{2} \\
& =(1-\cos s)\left|a_{1 s}\right|^{2} ;
\end{aligned}
$$

i.e., it is proportional to the number of atoms left in the ground state by the pump sequence, so providing a readout of the target qubit. If the probe beam arrives after a pair of $x$-polarized pump pulses then $a_{1 s}$ and $a_{2 p y}$ are given by Eq. (5) and if the probe is weak,

$$
\Delta \zeta=\frac{1}{2} s^{2}\left\{1-\frac{1}{2} \sin ^{2} S\left[1+\cos \left(\omega t_{d}\right)\right]\right\},
$$

which exhibits the same Ramsey fringes. Looking at the change in the amplitudes $\mathbf{B}-\mathbf{A}$, the amplitude for the $2 p_{x}$ state is completely unaffected, and the amplitude for the $1 s$ state is only affected to order $s^{2}$. In contrast, a direct probe with parallel polarization affects both qubit amplitudes to first order in $s$ (see Supplemental Material [15]). The weaker second-order effect raises the possibility of performing further coherent manipulation on the target qubit and continued probing of the state.

Here we utilize this readout to observe the Ramsey fringes produced by an equal pair of pump pulses, and extract the inhomogeneous dephasing time $T_{2}^{*}$. In this experiment, the first pump pulse rotates the Bloch vector about the $x$ axis. The second, parallel pulse rotates it again, this time with an axis of rotation at an azimuthal angle that depends on the delay time (but not on the spatial position). Thus the $z$ component of the Bloch vector produced by the pair of pumps oscillates, and this is observed by the average probe transmission just as discussed above.

\section{EXPERIMENT}

The sample was cleaved from a commercial float zonegrown natural silicon wafer with a $6 \times 10^{14} \mathrm{~cm}^{-3}$ phosphorus concentration. The edges of the sample are all parallel to $\langle 100\rangle$ directions and the sample dimensions are $10 \times 10 \times 0.5 \mathrm{~mm}$. The sample was mounted in vacuum on the cold finger of a

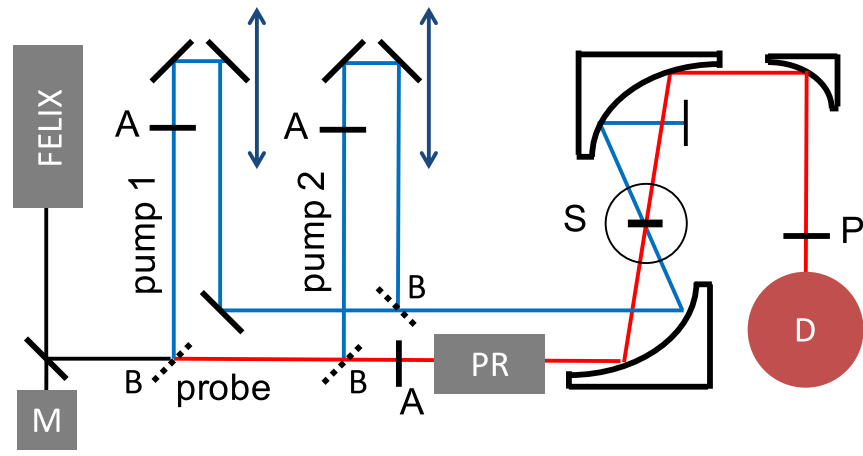

FIG. 2. Experimental setup. FELIX is the laser source, $M$ is a mononchomator behind a small hole near the edge of one mirror; A's are attenuators; delay stages for controlling the relative delays are shown by arrows; B denotes beam splitters; PR is a polarization rotator consisting of mirrors; $\mathrm{S}$ is the sample; $\mathrm{P}$ is a polarizer; $\mathrm{D}$ is the detector.

liquid helium cryostat with polypropylene film windows. The sample temperature was around $10 \mathrm{~K}$. For these conditions the optical linewidths of the transitions $1 s \rightarrow 2 p_{0}$ and $1 s \rightarrow 2 p_{ \pm}$ were measured by Fourier transform infrared interferometry (FTIR; see Supplemental Material [15]) and found to be $\Delta f_{A}^{(2 p 0)}=0.014 \mathrm{THz}$ and $\Delta f_{A}^{(2 p \pm)}=0.022 \mathrm{THz}$ at $4.2 \mathrm{~K}$, respectively (the resolution was $0.006 \mathrm{THz}$ ). The lines are inhomogeneously broadened and we do not expect any effect from the difference in temperature between FTIR and Ramsey experiments.

The experimental setup with the two parallel pump beams and a probe is shown in Fig. 2. Calibrated wire mesh attenuators (A) were used to ensure that both pump beams each produce $\pi / 2$ rotations on the Bloch sphere (taking the value of the dipole moment from [7]) within a tolerance of $10 \%$ (about $80 \mathrm{~nJ}$ per pulse). The polarization of the probe beam was rotated $90^{\circ}$ by a polarization rotator (RP) and a polarizer analyzer $(\mathrm{P})$ in front of the detector eliminated scattered light from the pump beams. The probe pulse area was about $\pi / 20$. The detector (D) was a helium cooled Ge:Ga photoconductor (signal output is proportional to intensity). The beam splitters (B) were polypropylene films with a reflection to transmission ratio close to 50:50 for $S$-polarized light at the frequency of interest. The Dutch Free Electron Laser (FELIX), which provides transform-limited pulses with controllable spectral bandwidth and pulse duration, was used as a light source. The output of the free-electron laser $(9.48 \mathrm{THz})$ was chosen to coherently excite the $1 s \rightarrow 2 p_{ \pm}$transition, as measured with a monochromator $(\mathrm{M})$. The time delay between the pulses [Fig. 3(a)] was controlled by stepper motor-driven delay stages. The delay between the probe and one of the pumps, labeled "pump 1," was fixed at 50 ps. This time was chosen because it is shorter than the population lifetime $\left(T_{1}=160 \mathrm{ps}[1]\right)$ and longer than the expected half width of the Ramsey fringes $\left(0.88 / 2 \Delta f_{A}^{(2 p \pm)}=20\right.$ ps where the factor 0.88 assumes the line is Gaussian in shape-see below). In this way the probe is sensitive to population produced by the pump, but not coherence. The arrival time of the other pump, labeled "pump 2," was varied relative to the other two pulses, as shown in Fig. 3(b). The result of the experiment 


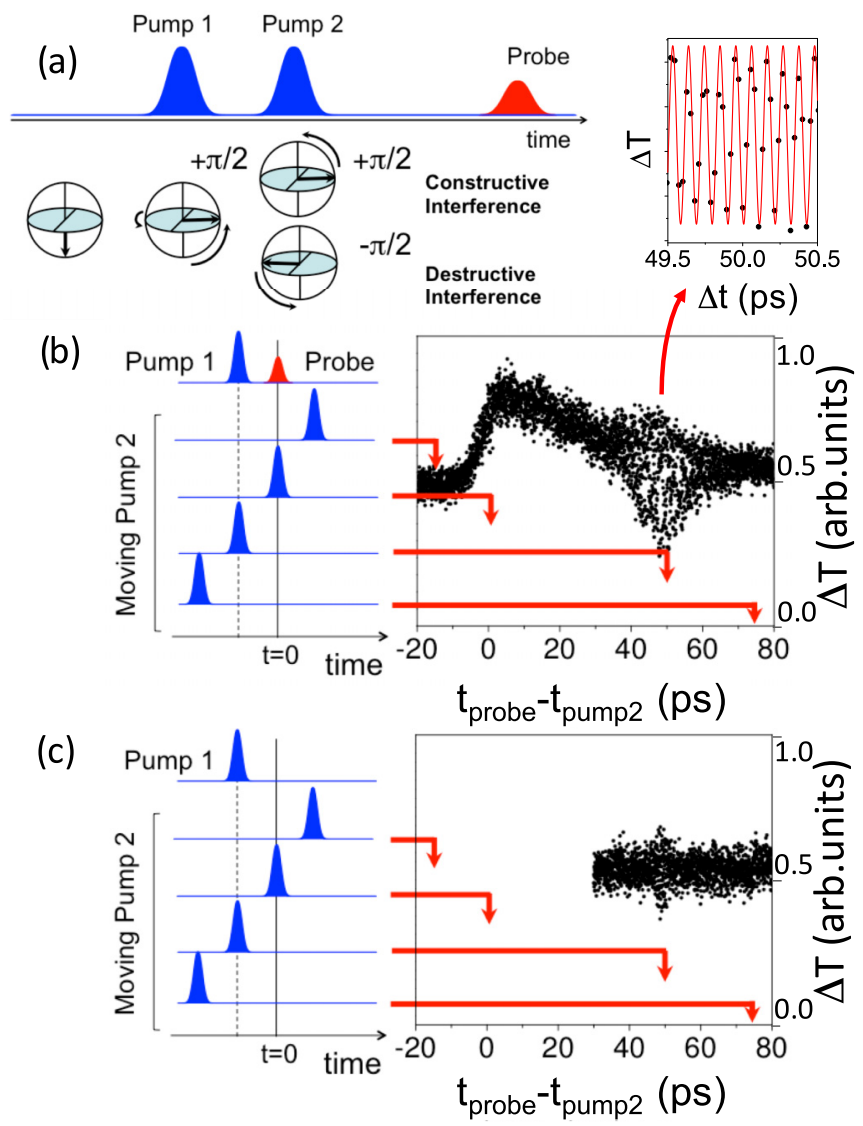

FIG. 3. The time-domain transmission. (a) Schematic of the pulse sequence and the effect of the two pumps on the Bloch sphere. The first pump performs a $\pi / 2$ rotation about the $x$ axis, and the Bloch vector precesses about the $z$ axis between pulses. The second pump performs a further rotation about the $x$ axis and depending on its phase relative to the oscillators this amplifies or destroys the wave packet. The probe transmission is sensitive to the population in the ground state. (b) The probe transmission change induced by the pumps $\Delta T=T-T_{0}$ where $T_{0}$ is the detected signal with both pumps blocked. The abscissa is the delay between one of the pumps and probe. The other pump arrives a fixed time of $50 \mathrm{ps}$ before the probe. An interference pattern is visible when the pumps overlap in time at $50 \mathrm{ps}$. (c) The detected signal with the probe blocked. Again, interference is observed when both pumps overlap.

is also shown in Fig. 3(b). Neither pump nor probe was modulated.

As a reference, we performed the same experiment when the probe beam was blocked, and the result is shown in Fig. 3(c). In this case, the signal observed by the detector is the light from the pump beams scattered due to surface roughness (of sample, mirrors, etc). In later experiments (to be published elsewhere) we also moved the detector to the transmitted pump beams, but a simple blocking of one beam is a less invasive reference experiment.

The FEL is a synchronously pumped pulsed laser, and the synchronism of the pump (the electron pulses from the rf linac), and the light pulse oscillating in the cavity may be easily controlled by adjusting the cavity length. Detuning away from synchronism lengthens the light pulse and narrows the bandwidth. In this investigation we used three different full width at half maximum (FWHM) FELIX bandwidths, determined from a Gaussian fit of the spectra measured by a grating monochromator: $\Delta f_{L}=0.078 \pm 0.001,0.127 \pm 0.002$, and $0.223 \pm 0.004 \mathrm{THz}$ [see Figs. 4(a)-4(c) and see below for corresponding pulse durations]. Note that these values are all significantly wider than the sample absorption line ( $\Delta f_{A}$ given above).

\section{TIME-DOMAIN RESULTS}

The fractional change in probe transmission shown in Fig. 3(b), $\Delta T / T$, is averaged over the beam profile-recall that this means it is averaged over phase and therefore measures population. When pump 2 arrives after the probe ( $t_{\text {probe }}<t_{\text {pump2 }}$ ), $\Delta T / T$ is defined only by the excitation created by pump 1 , resulting in a background level of around $\Delta T / T=12 \%$. When pump 2 arrives simultaneously or before the probe $\left(t_{\text {probe }} \geqslant t_{\text {pump } 2}\right)$ a transient contribution to $\Delta T / T$ is observed with a characteristic time $T_{1}=160 \mathrm{ps}$ due to relaxation of the extra population, in agreement with the decay time measured previously by traditional pump-probe experiments [1]. Apart from the regular pump-probe effect, there is an additional effect when $t_{\text {pump1 }} \approx t_{\text {pump2 }}\left(t_{\text {probe }}-\right.$ $\left.t_{\text {pump2 }} \approx 50 \mathrm{ps}\right)$, Fig. 3(b). Around this point in the transient the two pumps interfere, producing a rapidly oscillating population that is detected as an oscillation in $\Delta T / T$. The interference observed when the probe was blocked, Fig. 3(c), at $t_{\text {pump1 }} \approx t_{\text {pump2 }}$ is simply the linear correlation of the two pump pulses, i.e., the autocorrelation of the laser, due to stray reflections.

\section{FREQUENCY-DOMAIN ANALYSIS}

Data were analyzed in the frequency domain via evaluation of the fast Fourier transform (FFT) of the transient data shown in Figs. 3(b) and 3(c) over the time delay window from 20 to 80 ps where the interference between the pumps occurs. The results for all laser pulse bandwidths are shown in Fig. 4 by dots. The FFTs of the autocorrelation traces (i.e., probe blocked) are shown on Fig. 4(d,e,f), fitted by Gaussians. Note that the noise in the time-domain signal is Gaussian, and, therefore, so is the noise in its complex FFT, but the noise in the magnitude of the FFT has a Ricean distribution, which appears Gaussian for a large signal but is biased positively for a small signal. Therefore we used a simple least-squares fit, and forced the background to zero. The amplitudes and line centers were free parameters, whereas the FWHMs of each line were fixed to the corresponding laser bandwidths from the spectrometer measurement given above. Although the noise is strong because the origin of the signal is only weakly scattered light, the widths of the fits are in reasonable agreement with the widths of the peaks in the data, confirming that the fringes are due to the laser pulse autocorrelation.

The FFTs of the Ramsey fringes (probe unblocked), Figs. 4(g)-4(i), were fitted with Gaussians in the same way, except that the FWHM was a global free parameter, i.e., the same for all three experiments. The FWHM parameter extracted from the fitting was $\Delta f_{R}=0.0282 \pm 0.0004 \mathrm{THz}$. The point spacing of the FFT is determined by the inverse of the 60-ps delay window mentioned above, and was $0.017 \mathrm{THz}$ in this case, and though the error in the FWHM from the 

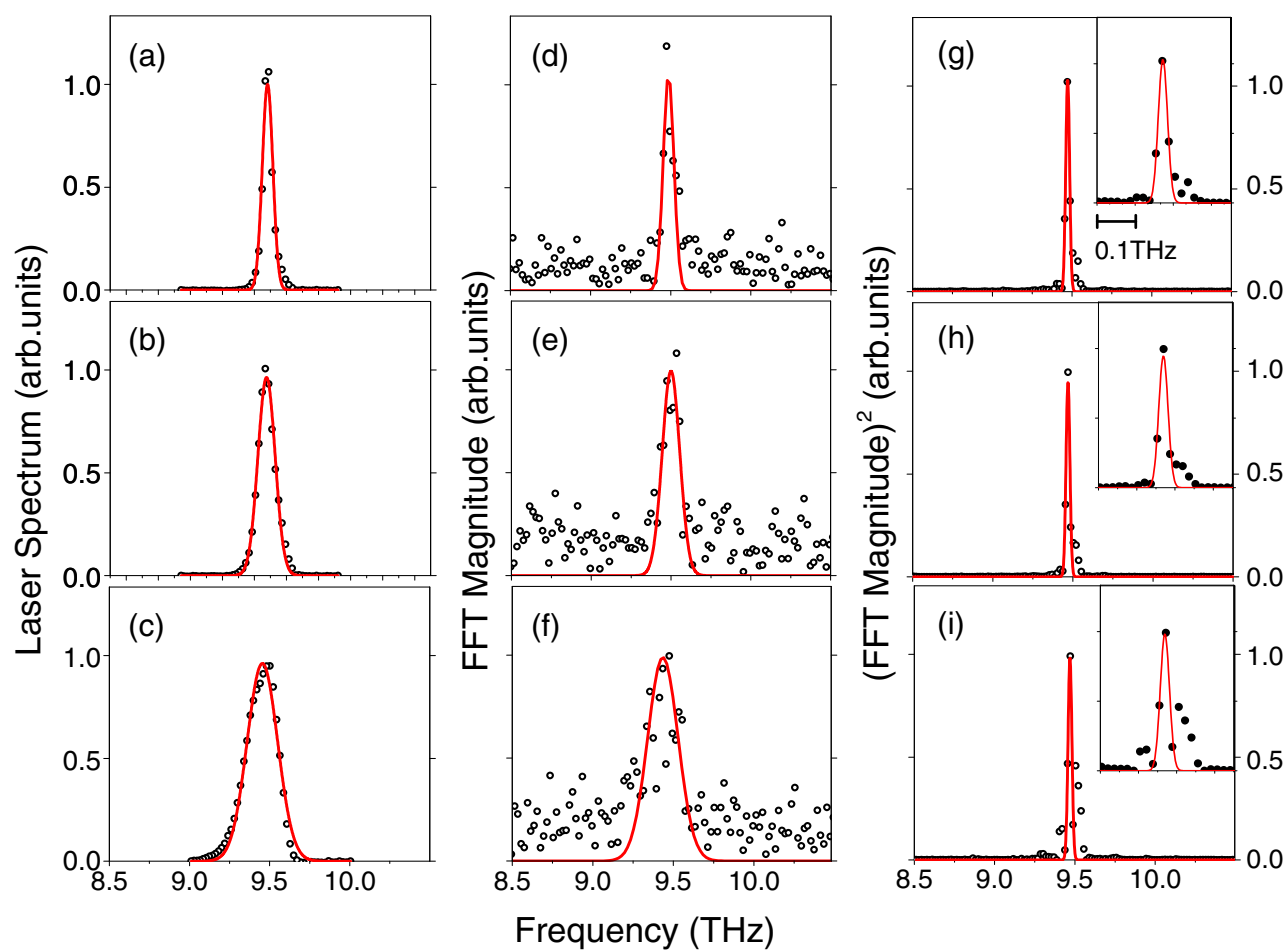

FIG. 4. Frequency-domain results for three different pulse-duration settings in three rows. Left column (a-c); the laser spectrum from a monochromator. Middle column (d-f); the corresponding magnitudes of the FFT of the laser autocorrelations [as Fig. 3(c)], proportional to the power spectrum of the laser. Right column (g-i); the magnitude squared of the FFT of the Ramsey fringes for each pulse-duration setting [as Fig. 3(b)], proportional to the absorption. The inserts are expanded versions of corresponding main panels. The solid lines in each panel are Gaussian fits (see text for explanation of fitting procedure). The width of the autocorrelation spectrum changes according to the laser bandwidth but the Ramsey fringes are much sharper in each case and unaffected.

least-squares fit was very much less, this explains the small difference between $\Delta f_{R}$ and the absorption linewidth from conventional, small signal FTIR, $\Delta f_{A}$ given above. Extending the range of delays and improving the signal to noise would improve the FFT point spacing and enable better agreement between the two. Note that in Figs. 4(g)-4(i) we used the magnitude squared of the FFT appropriate for the cross correlation of a short pulse with a long coherent oscillation. The noise is much less than for the autocorrelation experiment, partly because of the square but mainly due to the fact that this is a direct probe beam measurement rather than a weak scattering signal.

\section{TIME-DOMAIN ANALYSIS}

We also analyzed the data in the time domain to find the envelope of the fringes occurring at the laser frequency in the data of Fig. 3. It has been pointed out [16] that where time-domain quantities such as damping constants are the main objective, analysis in the frequency domain has disadvantages (such as the issue of Ricean noise). To do this here, we multiply the data by $\exp (i 2 \pi f t)$ where $f$ is the center frequency of the laser, smooth, and take the absolute magnitude. The effect is to produce the amplitude of fringes within the frequency window $f \pm \Delta f$ where $\Delta f$ is the inverse of the smoothing window time $\Delta t$. The results are shown in Fig. 5 (with $\Delta t=1 \mathrm{ps}$, chosen to be much smaller than $0.88 / \Delta f_{A}=40 \mathrm{ps}$ ) along with Gaussian fits. As for the frequency-domain fitting we forced the background to be zero in all cases, due to the Ricean noise. For the Ramsey fringes [Figs. 5(d)-5(f)] the FWHM of a simple Gaussian profile was a single global parameter, $\Delta \tau_{R}=20.6 \pm 0.2 \mathrm{ps}$. The autocorrelation time profiles [Figs. $5(\mathrm{a})-5(\mathrm{c})]$ were allowed unconstrained FWHMs, producing $\Delta \tau_{L}=10.4 \pm 0.2 \mathrm{ps}, 6.2 \pm$ $0.1 \mathrm{ps}$, and $3.4 \pm 0.1 \mathrm{ps}$. The errors quoted here for the values of $\Delta \tau_{L, R}$ are from the least-squares fits-note that the smoothing window was $1 \mathrm{ps}$, and a more realistic uncertainty for each is about 2 ps. More precision for $\Delta \tau_{L, R}$ could be gained by fitting directly the unfiltered fringe data [16] rather than fitting the envelope as we did here for illustrative purposes (Fig. 4).

\section{DISCUSSION}

In the case of a coherent laser pulse with a Gaussian envelope, the time-bandwidth product for the linear autocorrelation fringe duration (the FWHM of the envelope amplitude) and the FWHM of the intensity spectrum is $\Delta \tau_{L} \Delta f_{L}=4 \ln 2 / \pi=$ 0.88 [8]. Taking $\Delta \tau_{L}$ from the free fits of Fig. 5 and taking $\Delta f_{L}$ from the spectrometer measurement, we find $\Delta \tau_{L} \Delta f_{L}=0.81$, 0.79 , and 0.76 for the narrow, medium, and wide laser bandwidth cases, respectively. Taking into account the error in $\Delta \tau_{L}$ suggested above means that these values are indistinguishable from expectation. For very short laser pulses, the observed Ramsey fringe duration, $\Delta \tau_{R}$, is inversely proportional to the absorption linewidth $\Delta f_{A}$. We summarize the results for this work and others in Table I. In the case where the signal is 


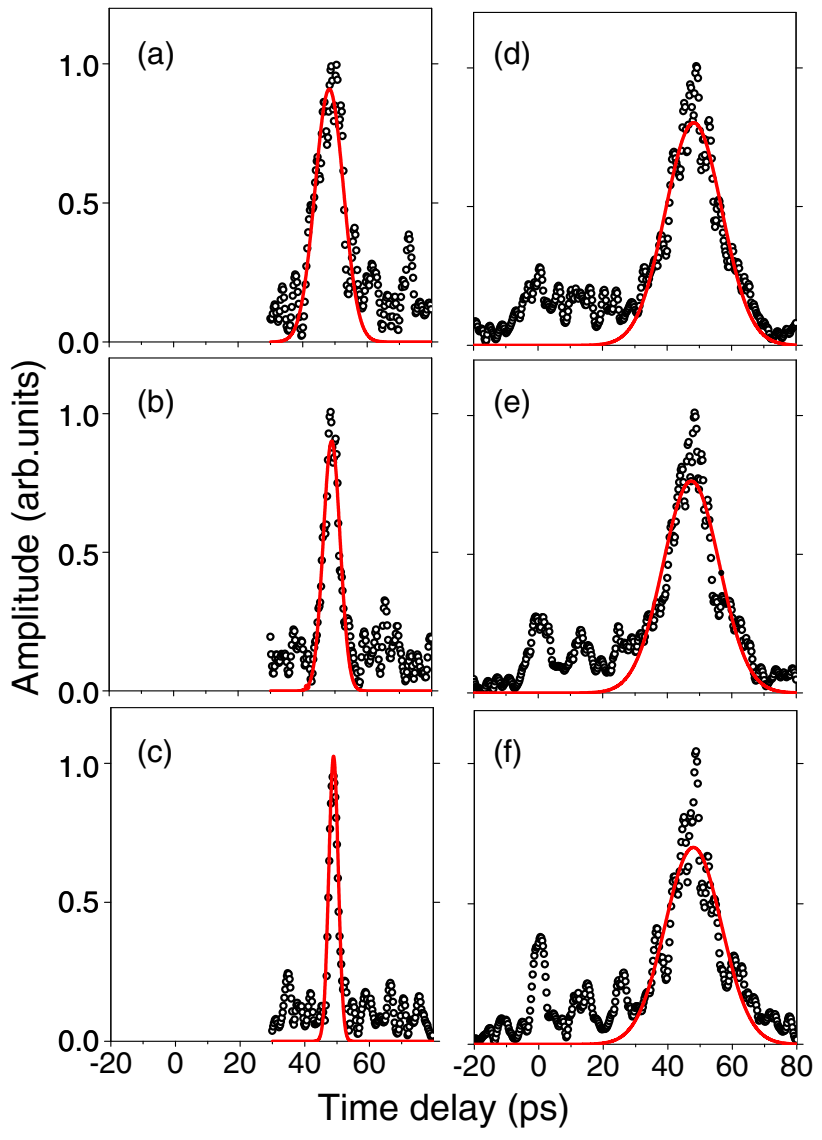

FIG. 5. The amplitude of the fringes in the data of Fig. 3 for three different laser pulse bandwidths shown in three rows. Left column (a-c) the amplitude of the autocorrelations [as Fig. 3(c)] which matches Figs. 4(d)-4(f), and right (d-f) the amplitude of the Ramsey fringes [as Fig. 3(b)] which matches Figs. 4(g)-4(i), along with fits (see text for explanation of the data processing and fitting procedure). The FWHM values for the fits on $(\mathrm{a}-\mathrm{c})$ were $\Delta \tau_{L}=$ $10.4 \pm 0.2 \mathrm{ps}, 6.2 \pm 0.1 \mathrm{ps}$, and $3.4 \pm 0.1 \mathrm{ps}$. The duration of the autocorrelation changes inversely with the laser bandwidth but the Ramsey fringe duration is unaffected.

proportional to either the excited-state population produced by a coherent Ramsey pair as in the previous electrical detection experiments [8,12], or to the ground-state population as in the present case of crossed-polarized probe transmission detection, $\Delta \tau_{R}^{\text {expect }}=4 \ln 2 / \pi \Delta f_{A}=0.88 / \Delta f_{A}$ for a Gaussian inhomogeneous line. In the case of echo detection $\Delta \tau_{R}^{\text {expect }}=4 \sqrt{2} \ln 2 / \pi \Delta f_{A}=1.25 / \Delta f_{A}$. In each case in Table I the measured time-bandwidth product deviates by
$2 \sigma$ or less from the expected value; i.e., it is therefore largely consistent with zero difference between observation and expectation. The agreement may be slightly improved in the two previous work cases ( 2 and 3 in Table I) by taking into account the convolution with the finite laser pulse. This lengthens the expected fringe duration; modeling suggests that to a good approximation $\Delta \tau_{R}^{\text {expect }}=\sqrt{x^{2} / \Delta f_{A}^{2}+0.88^{2} / \Delta f_{L}^{2}}$ for a Gaussian pulse where $x=0.88$ or 1.25 depending on the technique used. In the present case ( 1 in Table I), finite pulse-duration effects have been shown to be negligible (Fig. 5) and anyway would worsen agreement. This may be an indication that homogeneous dephasing has some effect. The homogeneous contribution to the linewidth is $0.001 \mathrm{THz}$ [3] to $0.002 \mathrm{THz}$ [7], and might be expected to be negligible for the samples used here, but it has been shown to increase with laser intensity [7]. For significant homogeneous dephasing the expected Ramsey fringe duration is shortened, and we have $1 / \Delta \tau_{R}^{\text {expect }}=\Delta f_{A} / x+\Delta f_{\text {hom }}$ where $\Delta f_{\text {hom }}=2 / T_{2}$ is the FWHM homogeneous linewidth. This would imply in the present measurement that $\Delta f_{\text {hom }}=0.024 \pm 0.008 \mathrm{THz}$. Note that this is quite an indirect estimate of the high-intensity $\Delta f_{\text {hom }}$, being the difference of inverse widths, and if small systematic errors were present they would have a large effect on the inferred result so it should be treated with caution. Such a laser-induced broadening would not have been noticeable in the echo experiment [7] which used a sample with a much larger inhomogeneous linewidth, and in the electrical experiment [8] the laser intensity was significantly lower (a pair of $\pi / 4$ pulses rather than a pair of $\pi / 2$ pulses, a quarter of the total energy). Note that the aim here is wider than estimation of $T_{2}$ or $T_{2}^{*}$-we have established the general crossed-polarized, weak probe measurement of the target qubit by showing that $\Delta \tau_{R}^{\text {expect }}-\Delta \tau_{R}^{\text {observed }} \approx 0$.

A relatively straightforward extension to this technique would allow direct measurement of $T_{2}$ by replacing the Ramsey pump sequence with a sequence consisting of a $\pi / 2$ pulse, $\tau_{12}$ wait; $\pi$ pulse, $\tau_{23}$ wait; and $\pi / 2$ pulse. This is the "three-pulse photon echo" sequence where the echo is projected by the final pulse to convert the polarization into population. The crossed-polarized probe beam then reads out the population as a function of the delay when $\tau_{12}=\tau_{23}=\tau$. In a conventional echo experiment (e.g., [7]) the signal is weak, and very difficult to find, especially in the $\mathrm{THz}$ regime where there is much diffraction scatter. In our proposed measurement of $T_{2}$ the signal is a change in the intensity of the probe beam, and the advantages relative to conventional echo are that even a small change in the intensity can be more detectable than the light emitted during the echo.

TABLE I. Comparison of absorption linewidths $\left(\Delta f_{A}\right)$ with Ramsey fringe durations $\left(\Delta \tau_{\mathrm{R}}\right)$ measured by different experimental techniques.

\begin{tabular}{llcrrr}
\hline \hline Sample & Detection & Transition & $\begin{array}{c}\Delta f_{A} \\
\text { THz }\end{array}$ & \multicolumn{1}{c}{$\begin{array}{c}\Delta \tau_{\mathrm{R}} \\
\text { ps }\end{array}$} & $\begin{array}{c}\Delta f_{A} \Delta \tau_{\mathrm{R}} \\
\text { Expt }\end{array}$ \\
\hline $1^{\mathrm{a}}$ & Pump probe & $1 s-2 p_{ \pm}$ & $0.022 \pm 0.006$ & $20.6 \pm 0.2$ & $0.45 \pm 0.15$ \\
$2^{\mathrm{b}}$ & Electrical & $1 s-2 p_{ \pm}$ & $0.028 \pm 0.006$ & $59 \pm 10$ & $1.65 \pm 0.63$ \\
$3^{\mathrm{b}}$ & Echo & $1 s-2 p_{0}$ & $0.046 \pm 0.006$ & $32.0 \pm 1.6$ & $1.47 \pm 0.26$ \\
\hline \hline
\end{tabular}

This paper.

${ }^{\mathrm{b}}$ Reference [8]. 


\section{CONCLUSION}

We have demonstrated a coherent dynamics experiment with readout performed by the transmission of an off-axis probe pulse. This is normally thought of as the geometry for incoherent experiments, but we have shown that it allows a perturbative readout of coherent qubit dynamics, analogous to quantum nondemolition. The technique may be extended simply to measure $T_{2}$, the homogeneous dephasing time. In a different application, the probe could also be used for full tomography of the target qubit. In this case a projection pulse is also required, this time with crossed polarization and propagation parallel to the pump beams. The technique is general and applies for any pulse shorter than the coherence time, and although we have applied it to a $\mathrm{THz}$ transition with a free-electron laser it opens the possibility for simplification of a range of coherent experiments with optical transitions and conventional short-pulse laser beams, for example, on molecular dynamics with mid-IR tabletop sources.

\section{ACKNOWLEDGMENTS}

This work was supported by the EPSRC-UK [COMPASSS/ADDRFSS, Grant No. EP/M009564/1], and the research programme of the Stichting voor Fundamenteel Onderzoek der Materie (FOM), which is financially supported by the Nederlandse Organisatie voor Wetenschappelijk Onderzoek (NWO). BNM is grateful for a Royal Society Wolfson Research Merit Award.

The raw data used in this work are available to download at https://doi.org/10.5281/zenodo.168373
[1] N. Q. Vinh, P. T. Greenland, K. Litvinenko, B. Redlich, A. F. G. van der Meer, S. A. Lynch, M. Warner, A. M. Stoneham, G. Aeppli, D. J. Paul, C. R. Pidgeon, and B. N. Murdin, Silicon as a model ion trap: time domain measurements of donor Rydberg states, Proc. Natl. Acad. Sci. USA 105, 10649 (2008).

[2] K. Saeedi, S. Simmons, J. Z. Salvail, P. Dluhy, H. Riemann, N. V. Abrosimov, P. Becker, H.-J. Pohl, J. J. L. Morton, and M. L. W. Thewalt, Room-temperature quantum bit storage exceeding 39 minutes using ionized donors in silicon-28, Science 342, 830 (2013).

[3] N. Steger, A. Yang, D. Karaiskaj, M. L. W. Thewalt, E. E. Haller, J. W. Ager III, M. Cardona, H. Riemann, N. V. Abrosimov, A. V. Gusev, A. D. Bulanov, A. K. Kaliteevskii, O. N. Godisov, P. Becker, and H.-J. Pohl, Shallow impurity absorption spectroscopy in isotopically enriched silicon, Phys. Rev. B 79, 205210 (2009).

[4] M. Cardona and M. L. W. Thewalt, Isotope effects on the optical spectra of semiconductors, Rev. Mod. Phys. 77, 1173 (2005).

[5] K. Saeedi, M. Szech, P. Dluhy, J. Z. Salvail, K. J. Morse, H. Riemann, N. V. Abrosimov, N. Notzel, K. L. Litvinenko, B. N. Murdin, and M. L. W. Thewalt, Optical pumping and readout of bismuth hyperfine states in silicon for atomic clock applications, Sci. Rep. 5, 10493 (2015).

[6] A. M. Stoneham, A. J. Fisher, and P. T. Greenland, Optically driven silicon-based quantum gates with potential for hightemperature operation, J. Phys.: Condens. Matter 15, L447 (2003).

[7] P. T. Greenland, S. A. Lynch, A. F. G. van der Meer, B. N. Murdin, C. R. Pidgeon, B. Redlich, N. Q. Vinh, and G. Aeppli, Coherent control of Rydberg states in silicon, Nature 465, 1057 (2010).

[8] K. L. Litvinenko, E. T. Bowyer, P. T. Greenland, N. Stavrias, J. Li, R. Gwilliam, B. J. Villis, G. Matmon, M. L. Y. Pang, B. Redlich, A. F. G. van der Meer, C. R. Pidgeon, G. Aeppli, and B. N. Murdin. Coherent creation and destruction of orbital wavepackets in $\mathrm{Si}: \mathrm{P}$ with electrical and optical read-out, Nat. Commun. 6, 6549 (2015).

[9] N. Q. Vinh, B. Redlich, A. F. G. van der Meer, C. R. Pidgeon, P. T. Greenland, S. A. Lynch, G. Aeppli, and B. N. Murdin, Time-Resolved Dynamics of Shallow Acceptor Transition in Silicon, Phys. Rev. X 3, 011019 (2013).

[10] E. T. Bowyer, B. J. Villis, J. Li, K. L. Litvinenko, B. N. Murdin, M. Erfani, G. Matmon, G. Aeppli, J.-M. Ortega, R. Prazeres, L. Dong, and X. Yu, Picosecond dynamics of a silicon donor based terahertz detector device, Appl. Phys. Lett. 105, 021107 (2014).

[11] K. L. Litvinenko, S. G. Pavlov, H.-W. Hubers, N. V. Abrosimov, C. R. Pidgeon, and B. N. Murdin, Photon assisted tunneling in pairs of silicon donors, Phys. Rev. B 89, 235204 (2014).

[12] P. T. Greenland, G. Matmon, B. J. Villis, E. T. Bowyer, J. Li, B. N. Murdin, A. F. G. van der Meer, B. Redlich, C. R. Pidgeon, and G. Aeppli, Quantitative analysis of electrically detected Ramsey fringes in P-doped Si, Phys. Rev. B 92, 165310 (2015).

[13] F. A. Zwanenburg, A. S. Dzurak, A. Morello, M. Y. Simmons, L. C. L. Hollenberg, G. Klimeck, S. Rogge, C. N. Coppersmith, and M. A. Eriksson, Silicon quantum electronics, Rev. Mod. Phys. 85, 961 (2013).

[14] P. Grangier, J. A. Levenson, and J.-P. Poizat, Quantum non-demolition measurements in optics, Nature 396, 537 (1998).

[15] See Supplemental Material at http://link.aps.org/supplemental/ 10.1103/PhysRevB.94.235207 for theoretical consideration of a three-level scheme pump-probe experiment and for a transmission spectrum of the sample under investigation.

[16] H. Barkhuijsen, R. de Beer, and D. van Ormondt, Improved algorithm for noniterative time-domain model fitting to exponentially damped magnetic resonance signals, J. Magn. Reson. 73, 553 (1987). 